\title{
Inhibition of cancer cell proliferation by 5-fluoro-2'-deoxycytidine, a DNA methylation inhibitor, through activation of DNA damage response pathway
}

\author{
Quanyi Zhao, Jiadong Fan, Wei Hong, Lianyun Li ${ }^{*}$ and Min Wu
}

\begin{abstract}
Multiple epigenetic changes, including alterations in DNA methylation occur during tumorigenesis. Various inhibitors of DNA methylation have been developed to prevent proliferation of cancer cells. 5-fluoro-2'deoxycytidine (FCdR) is one such DNA methylation inhibitor, which is currently in phase II clinical trial. To investigate the molecular mechanism/s by which FCdR might mediate repression of tumor cell proliferation, we analyzed the toxicity of FCdR in various cell lines established from different sarcomas. We found HCT116, a colon cancer cell line, is much more sensitive to FCdR compared to others. FCdR treatment inhibited HCT116 cells at G2/ $M$ check point and up-regulated expression of multiple cancer-related genes, which could be due to its inhibitory activity towards DNA methylation. Furthermore, we found that FCdR activates DNA damage response pathway. Using an inhibitor for ATM and ATR kinases activity, which are required for amplifying the DNA damage repair signal, we show that FCdR induced inhibition of HCT116 cells at G2/M is mediated through activation of DNA damage response pathway.
\end{abstract}

Keywords: FCdR, DNA methylation, DNA damage response, Cell cycle, p53, Cancer

\section{Background}

DNA methylation is a covalent modification of methyl group on the $5 \mathrm{C}$ site of cytosine nucleoside (Robertson 2005; Ren et al. 2011) and is dynamically regulated by methylation and demethylation (Carey et al. 2011). High level of DNA methylation on gene promoters is associated with transcriptional repression of genes. During carcinogenesis, global levels of DNA methylation decrease along with progression of cancer. Concomitantly, promoters of tumor suppressors gain DNA methylation, which allow cancer cells to grow unrestrained (Robertson 2005; Esteller 2008; Ellis et al. 2009). These observations have led to the development of small molecule inhibitors capable of inhibiting DNA methylation. These are thought to suppress tumorigenesis by activating the expression of tumor suppressor genes. Some of these DNA methylation inhibitors, such as Vidaza (5-azacytidine, 5-azaC) and

\footnotetext{
* Correspondence: lilianyun@whu.edu.cn; wumin@whu.edu.cn

Hubei Clinical Centre \& Key Laboratory of Intestinal and Colorectal Diseases, and College of Life Sciences, Wuhan University, Wuhan, Hubei 430072, China
}

Decitabine (5-aza-2'-deoxycytidine, 5-azaCdR) have been approved by FDA for treatment of myelodysplatic syndrome (Ellis et al. 2009; Ren et al. 2011). Although many other non-nucleoside DNA methylation inhibitors have been synthesized, their activities in inhibiting DNA methylation and gene activation are relatively weaker and their potential use in clinics still needs to be investigated (Chuang et al. 2005). 5fluoro-2'-deoxycytidine (FCdR, FdCyd) is a wellknown DNA methylation inhibitor discovered in early 1990's and is currently under evaluation in clinical trials of breast cancer and other advanced solid tumors (Gowher and Jeltsch 2004; Ellis et al. 2009; Ren et al. 2011).

Like Vidaza and Decitabine, FCdR is a pyrimidine analogue and can integrate into chromatin, and inhibit DNA methylation (Ellis et al. 2009). Fluorine occupies the $5 \mathrm{C}$ site of cytidine, which prevents the modification by methyl group (Ren et al. 2011). Additionally, it was demonstrated that FCdR is capable of binding and 
trapping DNA methyltransferases, and thus can prevent further DNA methylation (Jones and Taylor 1980; Reither et al. 2003). FCdR was found to be not stable in multiple clinical studies (Beumer et al. 2006), but when combined with other drugs, such as tetrahydrouridine (THU) and dihydro-5-azacytidine (DHAC), FCdR showed increased stability and improved activity (Beumer et al. 2008; Kratzke et al. 2008). However, the molecular mechanism of repression of tumor suppression by FCdR has not been studied in any detail.

Upon treatment with DNA methylation inhibitors, tumor suppressor genes are activated, which then lead to cell cycle arrest or apoptosis. p53 is one of the best characterized tumor suppressor gene, mutated in up to $50 \%$ of cancers (Royds and Iacopetta 2006). p53 can be activated by various signals, such as irradiation or chemical induced DNA damage, abnormal oncogene expression, microtubule inhibitors and other stress conditions (Royds and Iacopetta 2006; Kruse and Gu 2009). Upon activation, p53 is phosphorylated and dissociated from MDM2, which results in its stabilization (Wade et al. 2010). Activated p53 transcribes a number of genes to induce cell cycle arrest, apoptosis, and senescence, all of which help in suppressing tumorigenesis (Vousden and Prives 2009; Speidel 2010; Aylon and Oren 2011).

Activation of DNA damage response is one of the most important mechanisms that represses tumorigenesis (Lord and Ashworth 2012). Malignancy of tumor is frequently associated with damage to chromatin, recombination and translocation (Schar 2001). Upon DNA damage, H2AX is phosphorylated by ATM, ATR or DNAPK at the DNA repair sites (Bonner et al. 2008). Phosphorylated H2AX further recruits the above kinases to the damaged foci, which results in amplification of the DNA damage signal (Bonner et al. 2008). ATM and ATR then phosphorylate CHK1, CHK2 and other molecules involved in DNA damage response to arrest cell cycle (Kastan and Lim 2000; Cimprich and Cortez 2008).

In order to investigate the molecular mechanisms of tumor repression by FCdR, we studied its effect on cell fate, gene expression and activation of signaling pathways. We found that FCdR represses proliferation of HCT116 at IC50 between 0.025-0.05 $\mu \mathrm{M}$. FCdR induced cell cycle arrest at G2/M phase and activated both p53 signaling and DNA damage response pathways. Our results suggest that FCdR induced G2/M arrest and suppression of cancer cell proliferation is mediated through FCdR's role in activation of DNA repair pathway.

\section{Results and discussion}

\section{FCdR inhibits proliferation of multiple cancer cell lines}

FCdR is in phase II clinical trial for treatment of breast cancer and many solid tumors. In order to test if cancer cells other than breast cancer cells are sensitive to FCdR, we chose HCT116, HEPG2, U2OS and KYSE150 cell lines representing colorectal carcinoma, hepatocellular carcinoma, osteosarcoma and oesophageal squamous cell carcinoma, respectively. We treated these cells with a series of FCdR concentrations. Surviving cells after $72 \mathrm{~h}$ treatment were then used to assay by MTT assay. FCdR inhibited the proliferation of all the above cell lines, but to different degrees. HCT116 cells showed less than $10 \%$ survival rate with $1 \mu \mathrm{M}$ FCdR and IC50 was between 0.025-0.05 $\mu \mathrm{M}$ (Figure 1A). At the same $1 \mu \mathrm{M}$ FCdR concentration, the survival rates of HEPG2, U2OS and KYSE150 cells were about 40\%, 80\% and 30\%, respectively. The observations suggest that colorectal tumors might be more sensitive to FCdR, compared to hepatocellular carcinoma, osteosarcoma and oesophageal squamous cell carcinoma.

\section{HCT116 cells are more sensitive to FCdR than SAHA and 5-azaC}

Several small molecules inhibiting epigenetic processes have been developed with an ability to inhibit cancer cells. SAHA and 5-azaC are two such small molecule inhibitors that have been approved by FDA. We tested and compared the cyto-toxicity of FCdR with SAHA and 5 -azaC on HCT116 cells, as well as one novel identified H3K9 methylation inhibitor BIX01294. We found that all the drugs tested repressed the proliferation of HCT116, however, their IC50 differed considerably (Figure 1B). IC50 of FCdR was lowest between 0.025$0.05 \mu \mathrm{M}$, whereas for 5-azaC, BIX01294 and SAHA, it was $5 \mu \mathrm{M}, 1.5 \mu \mathrm{M}$ and $0.25 \mu \mathrm{M}$ respectively. These findings suggested that HCT116 is much more sensitive to FCdR compared to SAHA and 5-azaC, which may prove to be of value in a clinical study.

\section{FCdR induces G2/M arrest in HCT116 cell}

Next we sought to study the effect of FCdR on cell cycle in HCT116 cells. Since drugs targeting DNA methylation are known to induce cell cycle arrest or apoptosis, we first performed cell cycle analysis by PI (Propidium Iodide) staining and analyzed cells with flow cytometry. Cells treated with $0.05 \mu \mathrm{M}$ FCdR for 48 h showed upto $24 \%$ of cells in G2/M phase (Figure 2A), whereas treatment with $0.5 \mu \mathrm{M}$ FCdR increased the percentage of cells in the G2M phase to $75 \%$ (Figure 2A). These results suggest that FCdR induces G2/M arrest in HCT116. To further substantiate our conclusion, we analysed the expression of cyclins by western blot (Figure 2B). Treatment with $0.5 \mu \mathrm{M}$ FCdR for $48 \mathrm{~h}$, resulted in significant increase in the total levels of cyclin B1.

Persistent cell cycle arrest leads to induction of apoptosis. However, HCT116 cells treated with FCdR at concentrations of up to $0.5 \mu \mathrm{M}$ for $48 \mathrm{~h}$, did not show any obvious apoptotic phenotype as observed by light 
A
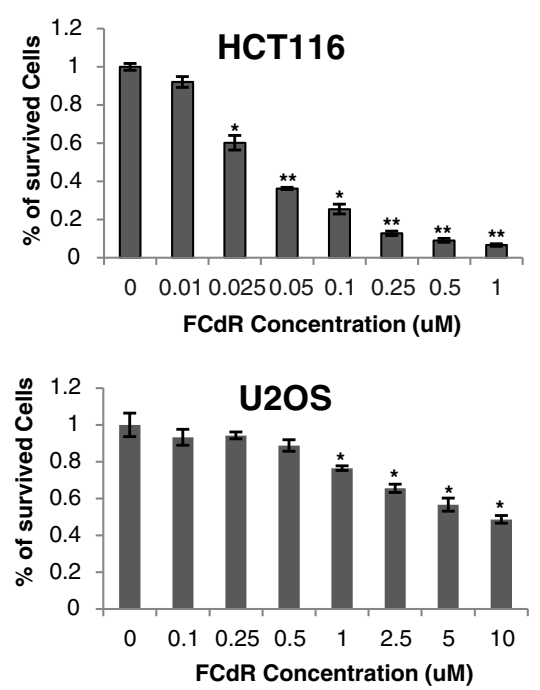

B
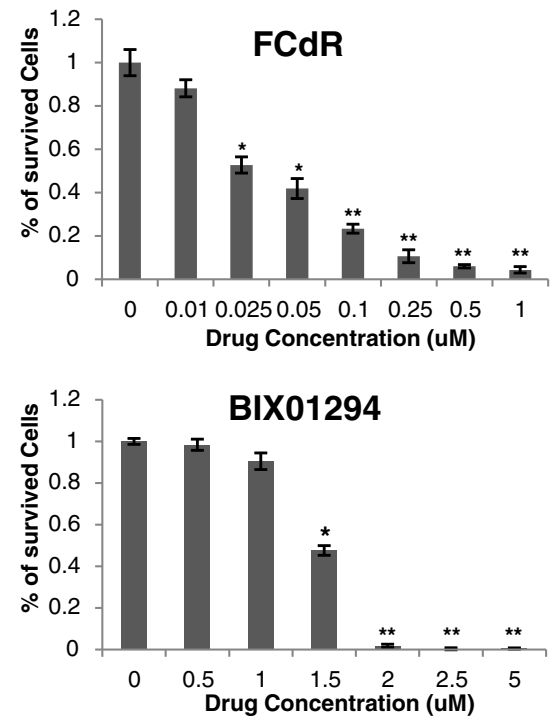
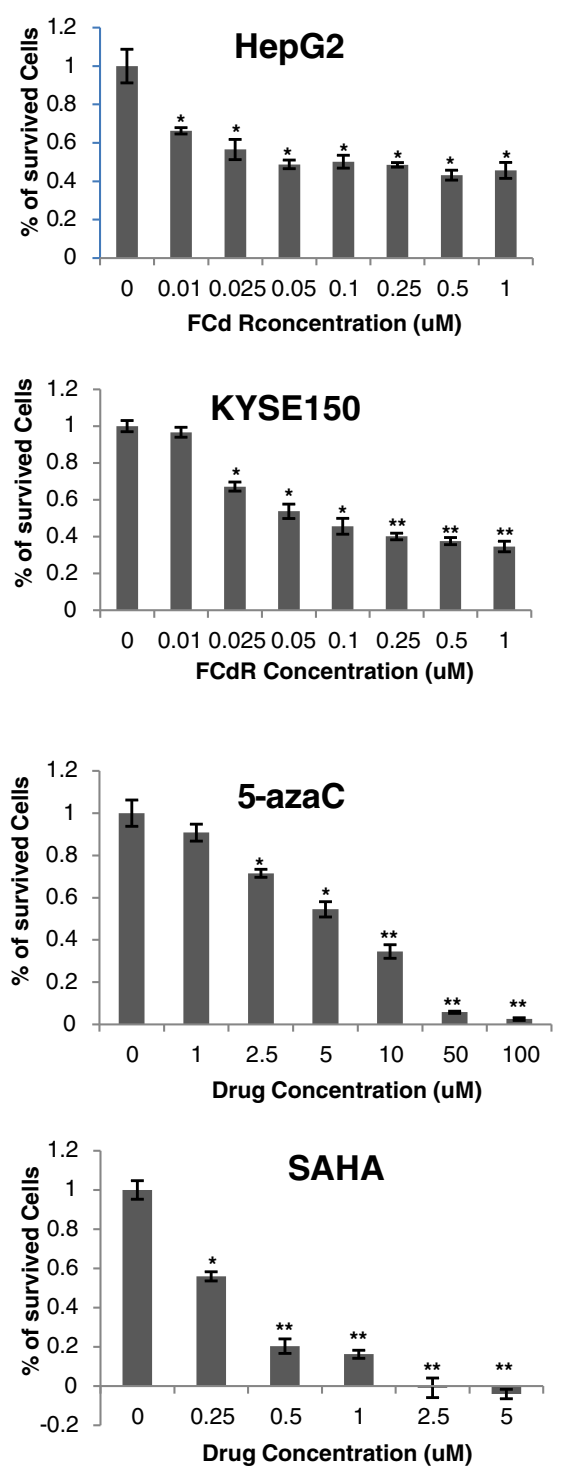

Figure 1 HCT116 cells are sensitive to FCdR treatment. A. HCT116, HepG2, U2OS and KYSE150 cell lines were treated with FCdR at indicated concentrations for $72 \mathrm{~h}$ and MTT assays performed. HCT116 cells were the most sensitive to FCdR. B. Four inhibitors against epigenetic enzymes were used to treat HCT116 cells for $72 \mathrm{~h}$, and MTT assay performed to test their toxicity. HCT116 cells were more sensitive to FCdR and SAHA, compared to 5-azaC and BIX01294. (* p value <0.05; ** $p$ value $<0.01$ ).

microscopy (data not shown). Flow cytometry analysis of these cells also did not show any obvious sub-G1 peak, which is a characteristic of apoptotic cells (Figure 2A). We further examined the formation of cleaved CASP3 and cleaved PARP, which are hallmarks of apoptosis. We did not detect any cleaved CASP3 or cleaved PARP by western blot whereas 5FU treatment, which induces apoptosis in HCT116 cells, resulted in cleavage of CASP3 and PARP (Figure 2C). These observations suggested that at the given concentration $\mathrm{FCdR}$ solely induces G2/M arrest in HCT116 and not apoptosis.
FCdR alters gene expression pattern by elevating transcription level

DNA methylation at gene promoters represses transcriptional activation and its inhibitors up regulate expression of genes. To investigate the mechanism/s involved in FCdR-induced G2/M arrest, we performed genome wide RNA sequencing (RNA-seq) of HCT116 cells treated with or without FCdR for $24 \mathrm{~h}$ and analyzed the alterations in gene expression. We also performed a similar experiment with 5-Fluorouracil, a widely used chemotherapeutic drug which induces DNA damage and cell cycle arrest, and used the RNA- 
A

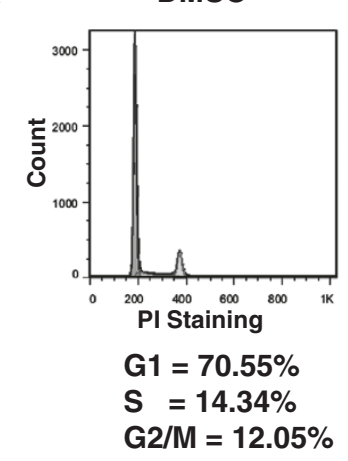

FCdR(0.05uM)

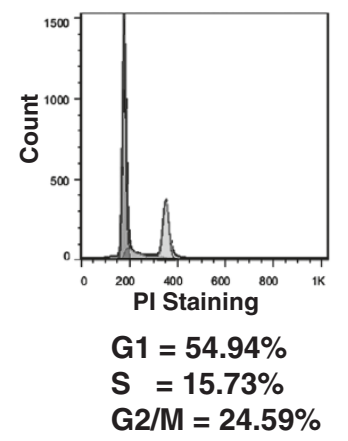

FCdR(0.5uM)

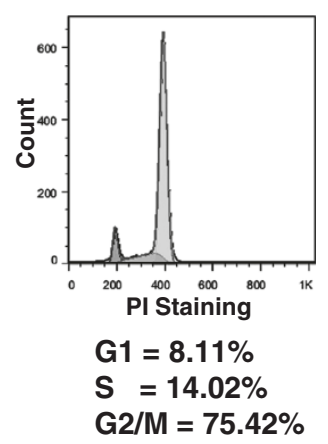

B
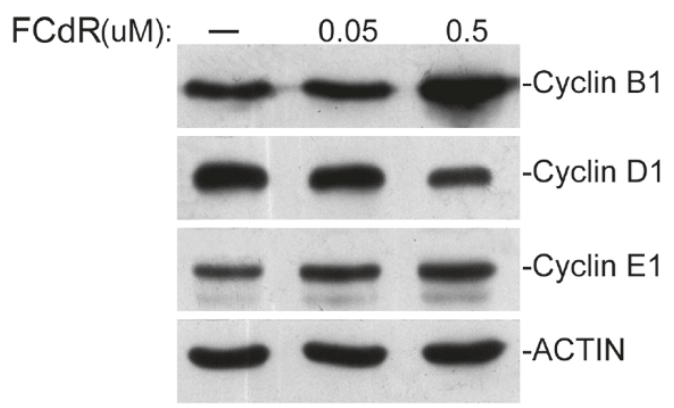

C

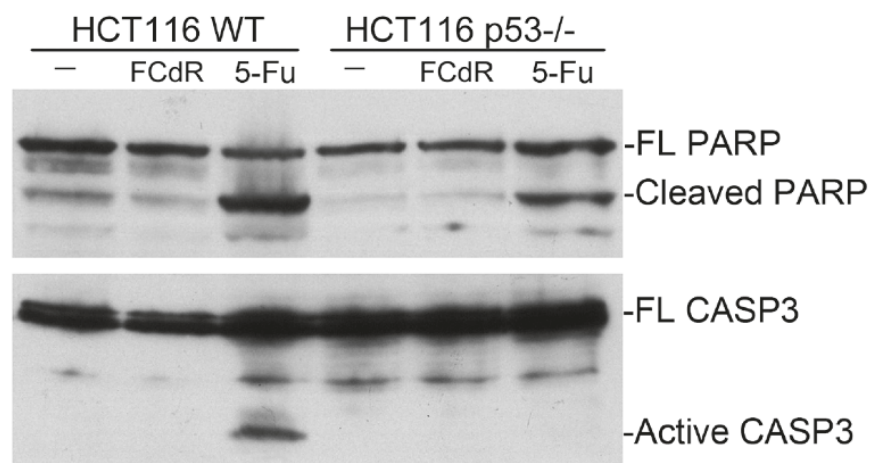

Figure 2 FCdR arrested HCT116 cells at G2/M phase. A. HCT116 cells were treated with $0.05 \mu \mathrm{M}$ or $0.5 \mu \mathrm{M}$ FCdR for $48 \mathrm{~h}$ and cell cycle was analyzed by flow cytometry. Compared with control, percentage of cells at G2/M phase increased significantly after FCdR treatment. B. HCT116 cells were treated with indicated amount of FCdR for $48 \mathrm{~h}$ and the cyclins checked by western blot. CyclinB1, which accumulates at G2/M phase, significantly increased after FCdR treatment. C. HCT116 p53+/+ and p53 $3^{-/-}$cells were treated with $0.5 \mu \mathrm{M} \mathrm{FCdR}$ or $375 \mu \mathrm{M} 5-\mathrm{Fu}$ for $48 \mathrm{~h}$ and apoptosis assayed by PARP and CASP3 western blot. 5-Fu treatment caused cleavage of PARP and CASP3, but not FCdR.

seq profile for comparison with FCdR dataset. To reduce background signals we only considered genes, expressions of which were changed by at least two fold. We found that FCdR treatment lead to alteration in expression of a total of 1165 genes, out of which 757 were up regulated and 408 were down regulated (Figure 3A and Additional file 1: Table S1). A higher number of up-regulated genes in FCdR treated cells is expected as FCdR is known to inhibit DNA methylation. In comparison, 5-Fu treatment resulted in change in expression of 3296 genes out of which, 2/3 were down regulated (Figure $3 \mathrm{~A}$ and Additional file 2: Table S2).
Next we looked at alterations of signaling pathways, and found many of them to be altered in cells treated with FCdR (Table 1). The pathways, which were significantly altered were also related with cancer, including p53 signaling, DNA repair, DNA replication, cell cycle (Table 1). We validated the altered expression of 45 genes involved in these pathways by reverse transcription followed by quantitative PCR (Figure 3C). We found that more than $90 \%$ of these genes were similarly altered as in our high throughput sequencing dataset.

We performed cluster analysis of differentially expressed genes involved in pathways, which were altered the most, including- p53 signaling pathway, 
A

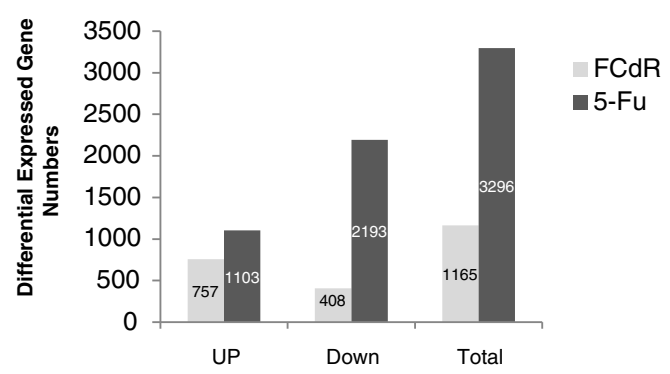

B

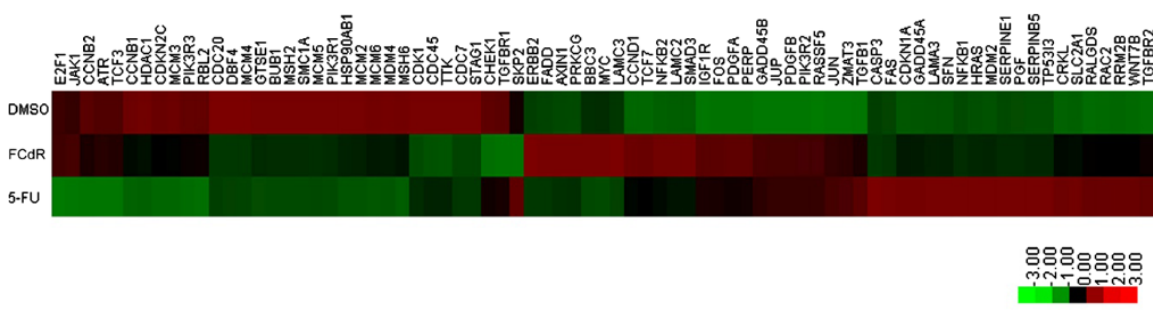

C

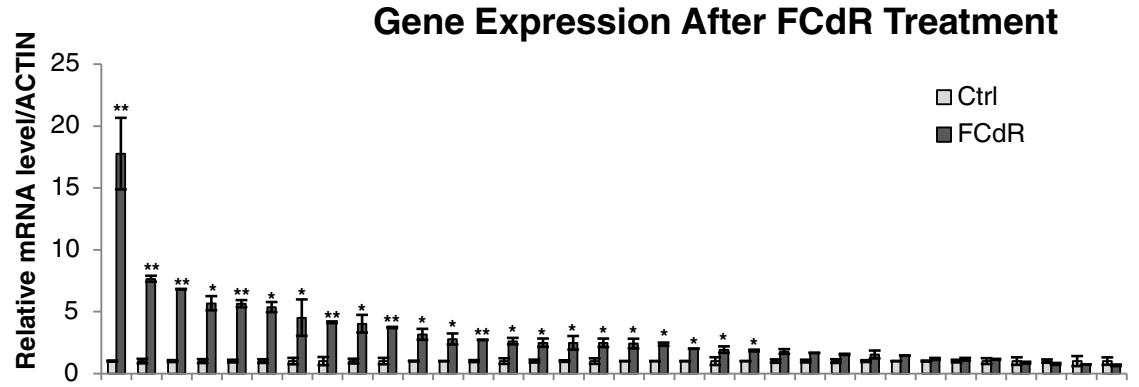

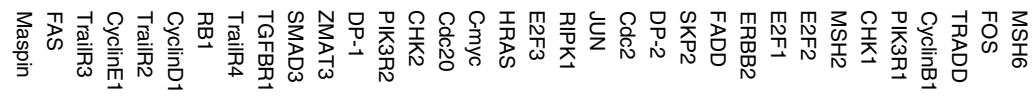

Figure $3 \mathrm{FCdR}$ induced alteration of global gene expression pattern. $0.5 \mu \mathrm{M} \mathrm{FCdR}$ or $375 \mu \mathrm{M}$ 5-Fu were used to treat HCT116 cells for $24 \mathrm{~h}$. Total RNA was used for gene expression pattern analysis by high throughput sequencing. A. The number of differentially expressed genes was calculated. FCdR caused changes in expression of 1165 genes, out of which 757 were up-regulated and 408 were down-regulated. 5-Fu caused changes in expression of 3295 genes, out of which 1103 were up-regulated, and 2193 were down-regulated. FCdR-treated cells have a much higher percentage of up-regulated genes. B. Differentially expressed genes involved in top 6 pathways (p53 signaling pathway, colorectal cancer, nucleotide excision repair, DNA replication, cell cycle, pathways in cancer) were subjected to cluster analysis. $\mathbf{C}$. The change in expression of 45 genes was confirmed by real time PCR. (* $p$ value $<0.05$; ** $p$ value $<0.01$ ).

colorectal cancer, nucleotide excision repair, DNA replication, cell cycle, pathways in cancer. We observed that both FCdR and 5-Fu treatment lead to similar changes in genes involved in DNA replication, DNA damage repair and p53 pathway (Figure 3B). Expression of a number of genes involved in DNA replication and repair were reduced in cells with both drugs. p53 target genes such as MDM2, CDKN1A/p21, SFN/14-3-36, and SERPINE1/PAI were also found to be activated in both samples, though in comparison to $\mathrm{FCdR}, 5-\mathrm{Fu}$ treatment resulted in stronger up-regulation of these p53 targets (Figures 3B and 4A). Among the genes up-regulated by FCdR, we also found several well-known proto-oncogenes, such as HRAS, CMYC and ERBB2 (Figure 3B and $C$ ). Increased expression of these genes might have implications in cancer treatment. Interestingly, we also observed that the receptor of TRAIL, TRAILR2, and the two decoy receptors, TRAILR3 and TRAILR4, were overexpressed (Figure 3C). TRAIL is a potential drugable protein which is known to induce apoptosis in many cancer cell lines but not in normal cells. It will be interesting to look at the effect of cancer treatment combining FCdR with TRAIL.

\section{FCdR treatment activated p53 signaling pathway} in HCT116

Our gene expression analysis of FCdR treated HCT116 cells suggest that FCdR activates p53 signaling pathway, which is the most important pathway inhibiting tumorigenesis. We further tested and confirmed the activation 
Table 1 Top 20 pathways altered in FCdR treated cells

\begin{tabular}{|c|c|c|c|c|c|c|}
\hline$\#$ & Pathway & $\begin{array}{l}\text { DEGs with pathway } \\
\text { annotation (594) }\end{array}$ & $\begin{array}{l}\text { All genes with pathway } \\
\text { annotation (9952) }\end{array}$ & Pvalue & Qvalue & Pathway ID \\
\hline 1 & p53 signaling pathway & $16(2.69 \%)$ & $67(0.67 \%)$ & $1.38 \mathrm{E}-06$ & $1.56 \mathrm{E}-04$ & ko04115 \\
\hline 2 & Colorectal cancer & $14(2.36 \%)$ & $62(0.62 \%)$ & $1.26 \mathrm{E}-05$ & $9.54 \mathrm{E}-04$ & ko05210 \\
\hline 3 & Nucleotide excision repair & $12(2.02 \%)$ & $48(0.48 \%)$ & $1.75 \mathrm{E}-05$ & $9.94 \mathrm{E}-04$ & ko03420 \\
\hline 4 & DNA replication & $10(1.68 \%)$ & $36(0.36 \%)$ & $3.28 \mathrm{E}-05$ & $1.49 \mathrm{E}-03$ & ko03030 \\
\hline 5 & Cell cycle & $20(3.37 \%)$ & $128(1.29 \%)$ & $6.57 \mathrm{E}-05$ & $2.49 \mathrm{E}-03$ & ko04110 \\
\hline 6 & Pathways in cancer & $36(6.06 \%)$ & $323(3.25 \%)$ & 2.06E-04 & $6.00 \mathrm{E}-03$ & ko05200 \\
\hline 7 & $\begin{array}{l}\text { Limonene and pinene } \\
\text { degradation }\end{array}$ & $3(0.51 \%)$ & $3(0.03 \%)$ & $2.12 \mathrm{E}-04$ & $6.00 \mathrm{E}-03$ & ko00903 \\
\hline 8 & $\begin{array}{l}\text { Biosynthesis of secondary } \\
\text { metabolites }\end{array}$ & $26(4.38 \%)$ & $225(2.26 \%)$ & 8.97E-04 & $2.26 \mathrm{E}-02$ & ko01110 \\
\hline 9 & Metabolic pathways & 92 (15.49\%) & $1132(11.37 \%)$ & 1.07E-03 & $2.43 \mathrm{E}-02$ & ko01100 \\
\hline 10 & Benzoate degradation & $4(0.67 \%)$ & $9(0.09 \%)$ & $1.24 \mathrm{E}-03$ & $2.57 \mathrm{E}-02$ & ko00362 \\
\hline 11 & Mismatch repair & $6(1.01 \%)$ & $23(0.23 \%)$ & $1.86 \mathrm{E}-03$ & $3.26 \mathrm{E}-02$ & ko03430 \\
\hline 12 & Prostate cancer & $13(2.19 \%)$ & $88(0.88 \%)$ & $2.05 \mathrm{E}-03$ & $3.26 \mathrm{E}-02$ & ko05215 \\
\hline 13 & Cell cycle - yeast & $11(1.85 \%)$ & $68(0.68 \%)$ & $2.13 \mathrm{E}-03$ & $3.26 \mathrm{E}-02$ & ko04111 \\
\hline 14 & Bladder cancer & $8(1.35 \%)$ & $40(0.4 \%)$ & $2.16 \mathrm{E}-03$ & $3.26 \mathrm{E}-02$ & ko05219 \\
\hline 15 & Meiosis - yeast & $10(1.68 \%)$ & $59(0.59 \%)$ & $2.35 \mathrm{E}-03$ & 3.33E-02 & ko04113 \\
\hline 16 & Ribosome & $13(2.19 \%)$ & $90(0.9 \%)$ & $2.52 \mathrm{E}-03$ & 3.36E-02 & ko03010 \\
\hline 17 & Adherens junction & $11(1.85 \%)$ & $70(0.7 \%)$ & $2.70 \mathrm{E}-03$ & $3.41 \mathrm{E}-02$ & ko04520 \\
\hline 18 & Base excision repair & $7(1.18 \%)$ & $34(0.34 \%)$ & $3.41 \mathrm{E}-03$ & 4.04E-02 & ko03410 \\
\hline 19 & Naphthalene degradation & $2(0.34 \%)$ & $2(0.02 \%)$ & $3.56 \mathrm{E}-03$ & 4.04E-02 & ko00626 \\
\hline 20 & Focal adhesion & $22(3.7 \%)$ & 199 (2\%) & $3.76 \mathrm{E}-03$ & 4.06E-02 & ko04510 \\
\hline
\end{tabular}

of p53 pathway by RTPCR analysis of mRNA levels of p53 target genes. We tested 11 p53 downstream genes and found that all were significantly elevated in expression (Figure 4A). As the activation of p53 involves stabilization of p53 protein, we analysed and found that the amount of p53 protein significantly increased after FCdR treatment, combined with the discovery that multiple p53 target genes increased their expression, suggesting that FCdR probably activates p53 pathway (Figure 4B).

In order to investigate if p53 signaling pathway is responsible for cell cycle arrest caused by FCdR treatment, we performed FCdR treatment in a p53 kncokout HCT116 cell line. We first verified the absence of p53 protein in these cells by western blot (Figure 4C). These cells, when treated with FCdR at a concentration of $0.5 \mu \mathrm{M}$, did not activate p53 target genes, including GADD45A, GADD45B and 14-3-3 $\sigma$ (Figure 4D). To our surprise, FCdR was still able to induce G2/M arrest in these cells in the absence of p53 (Figure 4E). Compared with parental HCT116 cells (Figure 2A), these cells showed G2/M arrest and similar distribution profile of other phases of cell cycle Also, cyclin B1 accumulation was comparable to parental cells (Figure 5A). Taken together, above observations suggest that the G2M arrest observed in FCdR treated cells is not a consequence of activation of the $\mathrm{p} 53$ pathway.

\section{FCdR induces DNA damage response in HCT116}

Since FCdR is capable of integrating into chromatin, and our FCdR treatment of HCT116 cells activated transcription of genes involved in DNA repair (Table 1), we investigated if FCdR has a role in DNA damage response pathway. To assess it, we first performed alkaline comet assay, and found that HCT116 cells treated with a low concentration of $0.02 \mu \mathrm{M}$ FCdR for $12 \mathrm{~h}$ exhibited DNA damage similar with $100 \mu \mathrm{M} 5-\mathrm{Fu}$, and the extent of DNA breaks increases at increasing doses of FCdR (Figure 5A and B). We then tested for phosphorylation of H2AX, ATM and CHK1, which are hallmarks of activated DNA repair pathway, and occur early during the DNA repair response. Western blot results showed a dramatic increase in levels of phosphorylated H2AX, ATM and CHK1 in HCT116 cells treated with $0.5 \mu \mathrm{M}$ FCdR (Figure 5C). Immunofluorescent staining also showed accumulation of phosphorylated $\mathrm{H} 2 \mathrm{AX}$ in the nuclei of FCdR treated HCT116 cells (Figure 5D). Since it is well known that activation of DNA damage response causes cell cycle arrest, it is highly likely that 


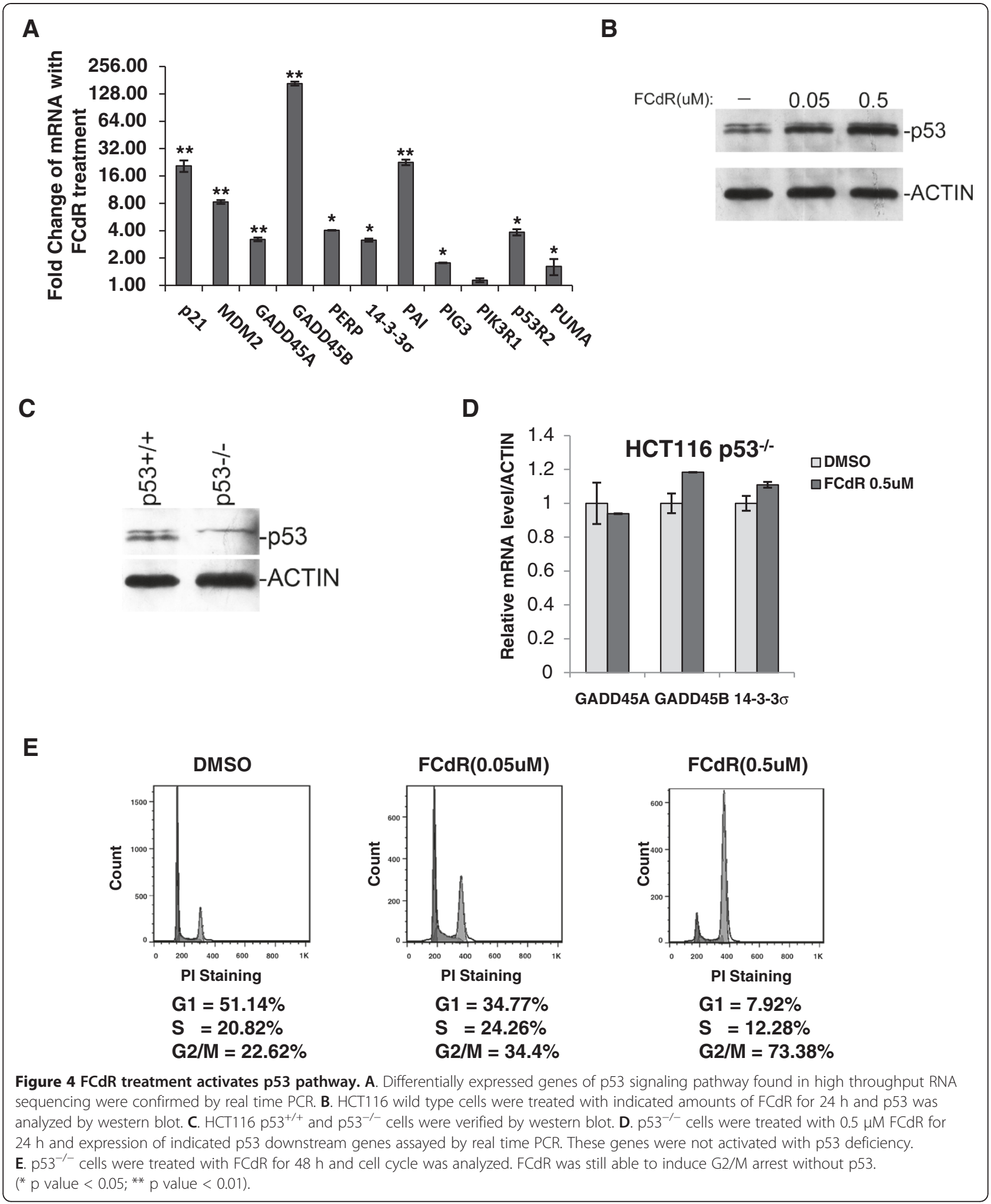

activation of DNA repair pathway is the primary reason of FCdR-induced cell cycle arrest.

To test if the induction of DNA damage response is a common feature for DNA methylation inhibitors, we treated HCT116 cells with various drugs, including two inhibitors of DNA methylation, FCdR and 5-azaC, and a histone deacetylase inhibitor SAHA. We observed that FCdR and 5-azaC treatment increased levels of 


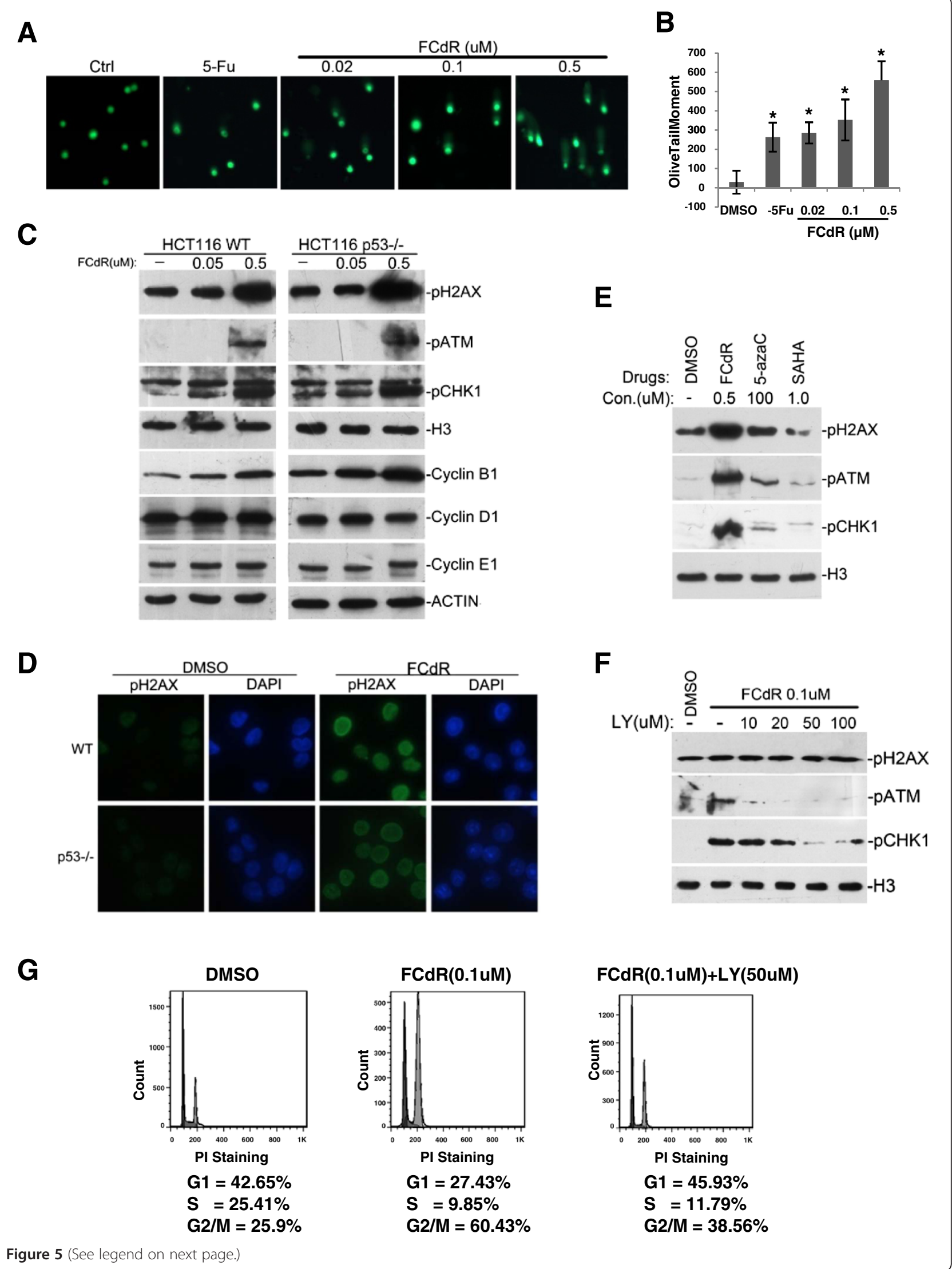


(See figure on previous page.)

Figure 5 DNA damage response is responsible for FCdR-induced cell cycle arrest. A. Cells were treated with indicated amount of chemicals for $12 \mathrm{~h}$ and damaged DNA was detected by alkaline comet assay. B. Olive tail moment in the previous assay was calculated according to manufacturer's method and the statistic results were shown. C. HCT116 $\mathrm{p} 53^{+/+}$and $\mathrm{p} 53^{-/-}$cells were treated with FCdR. Markers for DNA damage response (pH2AX, pATM and pCHK1) and cell cycle (Cyclin B1, Cyclin D1 and Cyclin E1) were analyzed by western blot. Histone H3 and $\beta$-ACTIN were used as loading controls. D. HCT1 $16 \mathrm{p} 53^{+/+}$and $\mathrm{p} 53^{-/-}$cells were treated with FCdR for $8 \mathrm{~h}$ and immunofluorescence staining was performed to show FCdR induced H2AX phosphorylation in both cell lines. E. Three drugs were used to treat HCT116 for $8 \mathrm{~h}$ and DNA damage responses were investigated by western blotting. FCdR and 5-azaC were able to induce phosphorylation of H2AX, ATM and CHK1, but not SAHA. H3 was used as control. F. The inhibitory effect of LY294002 to FCdR induced DNA damage response was assayed. G. Addition of $50 \mu M$ LY294002 restored the G2/M arrest induced by $0.1 \mu \mathrm{M} F C d R$. (* $p$ value $<0.05)$.

phosphorylated H2AX, ATM and CHK1, whereas SAHA treatment did not show a significant increase (Figure 5E). This indicated that at least two DNA methylation inhibitors, FCdR and 50azaC, are able to activate DNA damage pathway at the indicated concentration.

To confirm if DNA damage response is the primary reason for FCdR induced cell cycle arrest, we investigated if addition of a small molecule LY294002, an inhibitor of DNA damage response can suppress the activation of FCdR mediated DNA damage response pathway. LY294002 inhibits the activity of multiple PI3K kinases, including ATM and ATR, the two key kinases involved in DNA damage response. Various combinations of different concentrations of FCdR and LY294002 were tested.We found that at concentrations higher than $50 \mu \mathrm{M}$, LY294002 inhibits phosphorylation of ATM and CHK1 induced by $0.1 \mu \mathrm{M}$ FCdR (Figure $5 \mathrm{~F}$ ). We performed cell cycle analysis on cells treated with both FCdR and LY294002, and compared with cells treated only with FCdR. We found that G2/M arrest observed in cells treated with $0.1 \mu \mathrm{M}$ FCdR was completely abolished in cells treated additionally with DNA damage response inhibitor LY294002 (Figure 5G). This observation suggests that FCdR induced $G 2 / M$ arrest is mediated through activation of DNA damage response pathway.

\section{Conclusions}

The inhibitors of DNA methylation and histone deacetylation have shown similar curative effects and reduced toxicity, compared to traditional chemotherapy drugs in treatment of cancers. To speed up their use in cancer treatment, it is critical to elucidate the cellular response and molecular mechanisms of these drugs. FCdR is a promising drug in clinical trial. However, we know little about the kinds of tumors which are sensitive to FCdR and the molecular mechanisms of FCdR mediated suppression of tumorigenesis. We found that HCT116, a colon cancer cell line, was extremely sensitive to FCdR, which suggested that FCdR might be effective in treatment of certain types of colon cancer. FCdR inhibits HCT116 proliferation by arresting cell cycle at G2/M phase, without activating the apoptotic pathway. By global gene expression profiling we found that p53 signaling is activated upon FCdR treatment. Interestingly, FCdR induced cell cycle arrest was not dependent on the activation of p53 pathway. Many chemotherapy drugs induce death in cancer cells through p53 activation; however, since p53 is mutated in more than $50 \%$ cancers, the curative effects of chemotherapy drugs vary among patients. FCdR might be useful in treating tumors with mutation in p53 gene.

Our results show that FCdR treatment causes global changes in gene expression in HCT116 cells, which may help us better understand the molecular mechanisms of FCdR-induced cellular responses. Not only had we observed up regulation of tumor suppressor genes, such as p21 and PUMA, we also observed increase of HRAS and CMYC, two well known oncogene. It will be important to evaluate their roles in FCdR-induced response. Compared with 5-Fu, FCdR caused less genes to express differentially but a higher percentage of upregulated genes. The ability of FCdR to inhibit DNA methylation may explain the observation that most altered genes were upregulated in FCdR treated cells.

FCdR also activated DNA damage response pathway, possibly due to its ability to incorporate into chromatin. Since, an inhibitor of ATM/ATR kinases, LY294002, can rescue the cell cycle arrest induced by FCdR, it suggested the activation of ATM/ATR pathways is responsible for the observed cell cycle arrest. It is likely that FCdR inhibits cell growth primarily by activating the DNA damage response pathway. The activation of p53 is an important consequence of DNA damage response. FCdR induced cell cycle arrest is not dependent on p53 activation, which suggests other molecules downstream of DNA damage pathway might be responsible. Another inhibitor of DNA methylation, 5-azaC also induced DNA damage response, but not SAHA, an inhibitor of histone deacetylation. It will be interesting to investigate if DNA damage response is a common mechanism involved in growth inhibition caused by DNA methylation inhibitors.

\section{Materials and methods}

Cell lines, antibodies and reagents

FCdR, 5-azaC, 5-azaCdR and BIX01294 were purchased from Sigma. SAHA (Vorinostat) was purchased from 
Cayman. HCT116 and U2OS cells were purchased from ATCC. KYSE150 was purchased from Cell Bank of Chinese Academy of Medical Science. HepG2 was a gift from Dr. Jianguo Wu (Wuhan University). HCT116 p53 $3^{-/-}$cell was a gift from Dr. Pengfei Wang of Stowers Institute for Medical Research. The antibodies against Cyclin B1 (Epitomics), Cyclin D1 (Epitomics), Cyclin E1 (Epitomics), p-H2AX (Epitomics), p-ATM (Epitomics), p-CHK1 (Epitomics), $\beta$-ACTIN (CWBIO), CASP (Cell Signaling) and H3 (Abcam), were purchased from indicated companies. Rabbit anti-PARP was a gift from Dr. Xiaodong Zhang (Wuhan University). Rabbit antip53 was raised in our lab against purified full length protein. The PCR primers are given in Additional file 3: Table S3.

\section{MTT assay}

Cells were split at $1 \times 10^{3}$ cells per well in 96-well plate. After $24 \mathrm{~h}$ cells were treated with drugs and cultured for 72 h. $25 \mu \mathrm{g}$ MTT was then added to each well and cells incubated for $4 \mathrm{~h}$ at $37^{\circ} \mathrm{C}$. The medium with the formazan sediment was dissolved in 50\% DMF and 30\% SDS (pH4.7). The absorption was read at 570nM. P value was calculated by $t$ test.

\section{Cell cycle assay}

Cells were split at $2-3 \times 10^{5}$ cells per well in 6 -well plates. After 12-14 h cells were treated with drugs and cultured for $48 \mathrm{~h}$. Cells were harvested by $0.05 \%$ Trypsin-EDTA digestion and centrifuged after PBS wash. Cells were fixed overnight with $70 \%$ ethanol. Flow cytometry analysis was performed after PI staining $(50 \mathrm{ug} / \mathrm{mL})$ and RNase digestion $(100 \mathrm{ug} / \mathrm{mL})$ at $37^{\circ} \mathrm{C}$ for $30 \mathrm{~min}$.

\section{Western blot}

Approximately $2 \times 10^{6}$ Cells were lyzed in 200ul $1 \times$ SDS loading buffer () and boiled at $95^{\circ} \mathrm{C}$ for $10 \mathrm{~min}$. $5-10 \mu \mathrm{l}$ sample was loaded to SDS PAGE gel for each lane and the separated proteins were transferred to nitrocellular membrane. The membrane was blocked in 5\% milk and hybridized with indicated first antibody over night and second antibody for $1 \mathrm{~h}$ before developing.

\section{Immuno-fluorescence staining}

Cells were cultured on cover slips, washed twice with PBS and then fixed with chilled methanol. Cells were then washed three times with PBS and blocked in PBS with $1 \%$ BSA for $10 \mathrm{~min}$. Cells were incubated with primary and secondary antibodies for one hour, respectively. Samples were mounted with prolong anti-fade kit (Invitrogen) and observed on a fluorescent microscope.

\section{Reverse transcription and quantitative PCR}

Cells were scraped and collected by centrifugation. Total RNA was extracted with RNA extraction kit (Yuanpinghao) according to manufacturer's protocol. Approximately $1 \mathrm{ug}$ of total RNA was used for reverse transcription with a first strand cDNA synthesis kit (Toyobo). The amount of mRNA was assayed by quantitative PCR. $\beta$-actin was used to normalize the amount of each sample. Assays were repeated at least three times. Data shown were average values \pm SD of one representative experiment. P value was calculated by $t$ test.

\section{Alkaline comet assay}

OxiSelect Comet assay kit was purchased from Cell Biolabs and comet assay was performed according to the manufacturer's protocol. Briefly, cells were split at 2$3 \times 10^{5}$ cells per well in 6-well plate and cultured for $12 \mathrm{~h}$. Drugs were added to the medium and cells were treated for indicated time. Individual cells are mixed with molten agarose and then treated with lysis buffer and alkaline solution. Following electrophoresis, the samples were dried and stained with a DNA dye, then observed with fluorescent microscope. The tail length of each cell was measured manually and the tail DNA percentage was quantified by using Quantity One software (Bio-rad). Then the Olive tail moment was calculated according to the following formula: Tail DNA\% X Tail moment length, as suggested by provided manual. Data shown were average values \pm SD. P value was calculated by $t$ test.

\section{Next generation sequencing and data analysis}

The cells were treated with desired drugs for $24 \mathrm{~h}$ before collection. Total RNA was extracted and reverse transcribed. Then the cDNA were analyzed by BGI. To study the relationship of the differential expressed genes, the values of selected genes were submitted for cluster analysis by using Cluster3.0 and the heatmap was presented by Java Treeview.

\section{Additional files}

Additional file 1: Table S1. Differential Expressed Genes between FCdR-treated and Control Cells.

Additional file 2: Table S2. Differential Expressed Genes between 5-Futreated and control cells.

Additional file 3: Table S3. Information of primers used in the study.

Competing interests

The authors declare that they have no competing interests.

Authors' contributions

QZ performed most of the experiments. JF helped in some of the

fluorescence staining experiments. WH helped in some of the MTT assays. MW and LL directed the project. MW wrote the manuscript. All authors read and approved the final manuscript. 


\section{Acknowledgement}

We thank Dr. Man Mohan in Stowers Institute for Medical Research for editing the manuscript.

This work was supported by grants from the National Basic Research Program of China (973 Program, 2011CB504206, 2012CB518700), the National Natural Science Foundation of China to Min Wu (30971502 and 91019013) and Lianyun Li (30971501, 31200653 and 30921001), Program for New Century Excellent Talents in University to Min Wu (NCET-11-0410).

Received: 25 July 2012 Accepted: 27 November 2012

Published: 13 December 2012

\section{References}

Aylon Y, Oren M (2011) New plays in the p53 theater. Curr Opin Genet Dev 21:86-92

Beumer JH, Eiseman JL, Parise RA, Joseph E, Holleran JL, Covey JM, Egorin MJ (2006) Pharmacokinetics, metabolism, and oral bioavailability of the DNA methyltransferase inhibitor 5-fluoro-2'-deoxycytidine in mice. Clin Cancer Res 12:7483-7491

Beumer JH, Parise RA, Newman EM, Doroshow JH, Synold TW, Lenz HJ, Egorin MJ (2008) Concentrations of the DNA methyltransferase inhibitor

5-fluoro-2'-deoxycytidine (FdCyd) and its cytotoxic metabolites in plasma of patients treated with FdCyd and tetrahydrouridine (THU). Cancer Chemother Pharmacol 62:363-368

Bonner WM, Redon CE, Dickey JS, Nakamura AJ, Sedelnikova OA, Solier S, Pommier Y (2008) GammaH2AX and cancer. Nat Rev Cancer 8:957-967

Carey N, Marques CJ, Reik W (2011) DNA demethylases: a new epigenetic frontier in drug discovery. Drug Discov Today 16:683-690

Chuang JC, Yoo CB, Kwan JM, Li TW, Liang G, Yang AS, Jones PA (2005) Comparison of biological effects of non-nucleoside DNA methylation inhibitors versus 5-aza-2'-deoxycytidine. Mol Cancer Ther 4:1515-1520

Cimprich KA, Cortez D (2008) ATR: an essential regulator of genome integrity. Nat Rev Mol Cell Biol 9:616-627

Ellis L, Atadja PW, Johnstone RW (2009) Epigenetics in cancer: targeting chromatin modifications. Mol Cancer Ther 8:1409-1420

Esteller M (2008) Epigenetics in cancer. N Engl J Med 358:1148-1159

Gowher H, Jeltsch A (2004) Mechanism of inhibition of DNA methyltransferases by cytidine analogs in cancer therapy. Cancer Biol Ther 3:1062-1068

Jones PA, Taylor SM (1980) Cellular differentiation, cytidine analogs and DNA methylation. Cell 20:85-93

Kastan MB, Lim DS (2000) The many substrates and functions of ATM. Nat Rev Mol Cell Biol 1:179-186

Kratzke RA, Wang X, Wong L, Kratzke MG, Green MR, Vokes EE, Vogelzang NJ, Kindler HL, Kern JA (2008) Response to the methylation inhibitor dihydro-5-azacytidine in mesothelioma is not associated with methylation of p16INK4a: results of cancer and leukemia group B 159904. J Thorac Oncol 3:417-421

Kruse JP, Gu W (2009) Modes of p53 regulation. Cell 137:609-622

Lord CJ, Ashworth A (2012) The DNA damage response and cancer therapy Nature 481:287-294

Reither S, Li F, Gowher H, Jeltsch A (2003) Catalytic mechanism of DNA-(cytosine-C5)-methyltransferases revisited: covalent intermediate formation is not essential for methyl group transfer by the murine Dnmt3a enzyme. J Mol Biol 329:675-684

Ren J, Singh BN, Huang Q, Li Z, Gao Y, Mishra P, Hwa YL, Li J, Dowdy SC, Jiang SW (2011) DNA hypermethylation as a chemotherapy target. Cell Signal 23:1082-1093

Robertson KD (2005) DNA methylation and human disease. Nat Rev Genet 6:597-610

Royds JA, lacopetta B (2006) p53 and disease: when the guardian angel fails. Cell Death Differ 13:1017-1026

Schar P (2001) Spontaneous DNA damage, genome instability, and cancer-when DNA replication escapes control. Cell 104:329-332
Speidel D (2010) Transcription-independent p53 apoptosis: an alternative route to death. Trends Cell Biol 20:14-24

Vousden KH, Prives C (2009) Blinded by the light: the growing complexity of p53. Cell 137:413-431

Wade M, Wang YV, Wahl GM (2010) The p53 orchestra: Mdm2 and Mdmx set the tone. Trends Cell Biol 20:299-309

doi:10.1186/2193-1801-1-65

Cite this article as: Zhao et al: Inhibition of cancer cell proliferation by 5-fluoro-2'-deoxycytidine, a DNA methylation inhibitor, through activation of DNA damage response pathway. SpringerPlus 2012 1:65.

\section{Submit your manuscript to a SpringerOpen ${ }^{\circ}$ journal and benefit from:}

- Convenient online submission

- Rigorous peer review

- Immediate publication on acceptance

- Open access: articles freely available online

- High visibility within the field

- Retaining the copyright to your article

Submit your next manuscript at $\gg$ springeropen.com 